This item was submitted to Loughborough's Research Repository by the author.

Items in Figshare are protected by copyright, with all rights reserved, unless otherwise indicated.

\title{
The ordered Heusler alloy Pd2MnIn investigated by photoelectron
}

\section{spectroscopy}

PLEASE CITE THE PUBLISHED VERSION

http://dx.doi.org/10.1088/0953-8984/19/17/176217

PUBLISHER

(c) IOP Publishing Ltd

VERSION

AM (Accepted Manuscript)

LICENCE

CC BY-NC-ND 4.0

\section{REPOSITORY RECORD}

Cropper, Michael D., and D. Brown. 2019. "The Ordered Heusler Alloy Pd2mnin Investigated by Photoelectron Spectroscopy”. figshare. https://hdl.handle.net/2134/10744. 
This item was submitted to Loughborough's Institutional Repository (https://dspace.lboro.ac.uk/) by the author and is made available under the following Creative Commons Licence conditions.

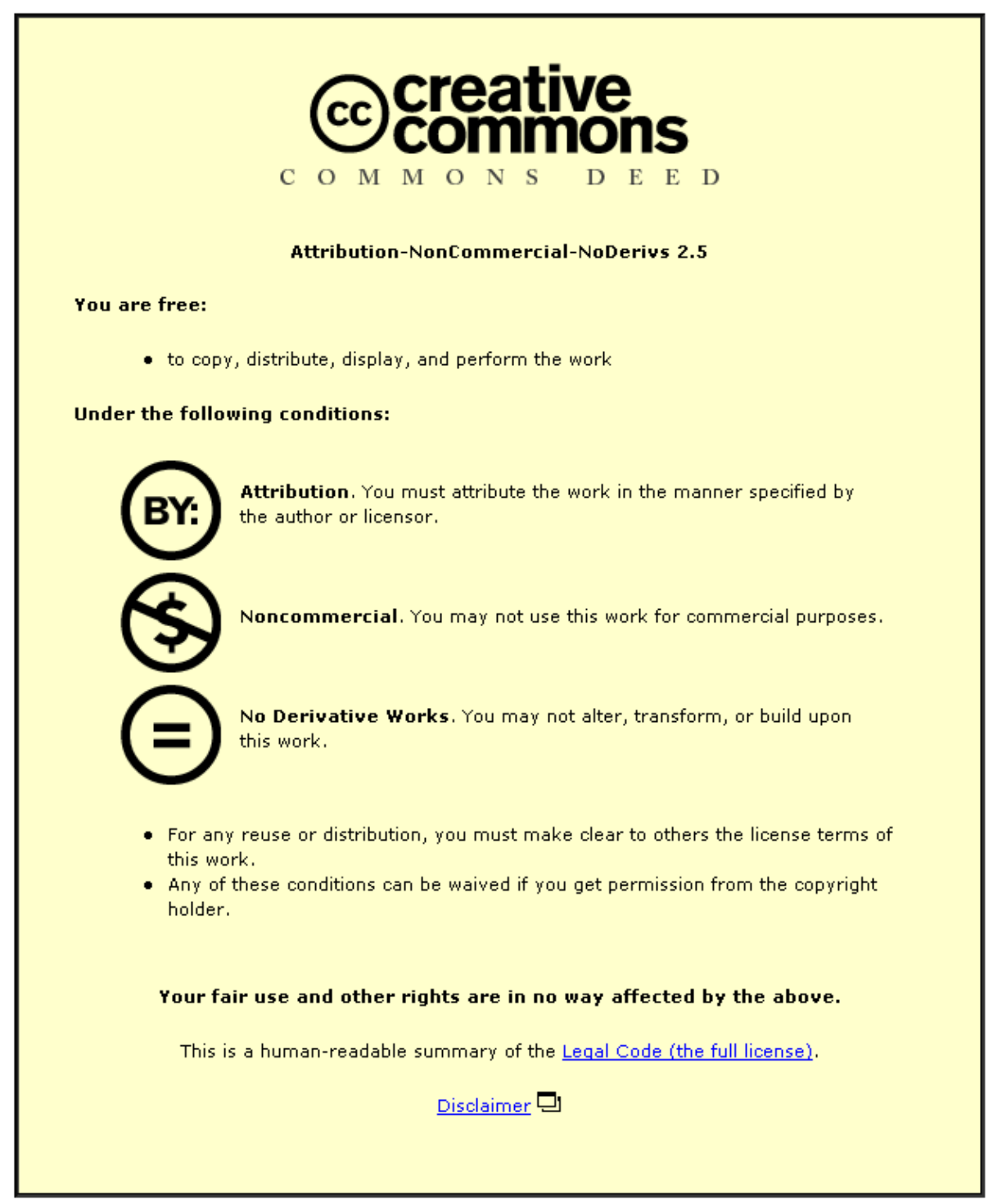

For the full text of this licence, please go to: http://creativecommons.org/licenses/by-nc-nd/2.5/ 


\title{
The ordered Heusler alloy $\mathbf{P d}_{2} \mathrm{MnIn}$ investigated by photoelectron spectroscopy
}

\author{
M D Cropper* and D Brown \\ Department of Physics, \\ Loughborough University, \\ Loughborough, \\ Leicestershire \\ LE11 3TU, \\ United Kingdom.
}

Author to whom correspondence should be addressed.

$$
\begin{gathered}
\text { Tel. +44 } 1509223308 \\
\text { Fax. +44 } 1509223986
\end{gathered}
$$

Email.M.d.cropper@lboro.ac.uk

Short title: Photoelectron spectroscopy of $\mathrm{Pd}_{2} \mathrm{MnIn}$

PACS Numbers 79.60, 71.20.-b, 73.61.At 


\section{ABSTRACT}

The full ordered antiferromagnetic Heusler alloy $\mathrm{Pd}_{2} \mathrm{MnIn}$ has been investigated using synchrotron radiation photoelectron spectroscopy. The Pd 4d Cooper minimum at 130 $\mathrm{eV}$ photon energy and the $\mathrm{Mn} 3 \mathrm{p}$ to $3 \mathrm{~d}$ resonance at $49.5 \mathrm{eV}$ photon energy were used to obtain information about the partial spectral weights due to the Pd 4d and Mn 3d derived states. These partial spectral weights were consistent with partial density of states published in the literature. A quantitative fit of the resonant profile yielded an asymmetry parameter of $1.6 \pm 0.4$, suggestive of largely delocalised Mn 3d states. 


\section{INTRODUCTION}

Heusler alloys ${ }^{1}$ have a long history of scientific investigation ${ }^{2}$ motivated by their huge variety of magnetic and other properties. Contemporary interest in their fabrication and properties is very large and expanding, fuelled by the discovery of properties that have potentially valuable technological applications including half-metallic behaviour $^{3}$ and ferromagnetic shape alloys ${ }^{4}$. The archetypal Heusler alloy is a structurally and chemically ordered compound of the form $\mathrm{X}_{2} \mathrm{MnZ}$ comprising four interpenetrating fcc lattices. The element $\mathrm{X}$ is usually a first row transition or noble metal such as $\mathrm{Co}, \mathrm{Ni}, \mathrm{Cu}, \mathrm{Pd}$ or Pt. Element $\mathrm{Y}$ is usually a group IIIB or IVB element such as Al, In, Sn or Sb. They are considered to be ideal arrays of magnetic moments localised on the Mn atoms. However, despite the localised magnetic behaviour, there is srtong evidence from band structure calculations ${ }^{5,6}$ supported by experimental evidence that the occupied Mn 3d states are delocalised. Calculations by Kübler et $\mathrm{al}^{6}$ using the augmented spherical wave method indicated that the Mn and X d electrons of the hybridised into a common band and that the localised moment was due to localisation of the unoccupied minority Mn 3d states.

$\mathrm{Pd}_{2} \mathrm{MnIn}$ is a weakly antiferromagnetic Heusler alloy with a moment of close to $4 \mu_{\mathrm{B}}$ confined to the $\mathrm{Mn}$ atoms ${ }^{7}$. It has been the subject of several experimental investigations including field and temperature dependent resistivity, Hall effect and magneto resistance ${ }^{8}$ and the influence of chemical order on the magnetisation properties $^{7,9}$. The evidence is that magnetic order is strongly dependent on chemical order, since as the structure disorders into the $\mathrm{B}_{2}$ structure, the minimum distance 
between Mn atoms decreases. The band structure of $\operatorname{Pd}_{2} \mathrm{MnIn}$ has been calculated ${ }^{6}$, but as yet there has been no direct experimental investigation of the band structure.

Synchrotron radiation excited ultraviolet photoemission spectroscopy has been widely used to probe the electronic structure of elements and alloys, and is a standard method for investigating the validity of band structure calculations ${ }^{10}$. In $\mathrm{Pd}_{2} \mathrm{MnIn}$ the valence band photoemission spectra will be dominated by transitions from the Mn 3d and Pd $4 \mathrm{~d}$ states since the photoionisation cross-sections for the $\mathrm{s}$ and $\mathrm{p}$ states are comparatively low. Hence, the valence band spectrum, $S\left(h v, E_{B}\right)$ can be described by:

$$
S\left(h v, E_{B}\right)=C(h v)\left[N_{P d} \sigma_{P d}(h v) D_{P d}\left(E_{B}\right)+N_{M n} \sigma_{M n}(h v) D_{M n}\left(E_{B}\right)\right]
$$

Where $h v$ is the photon energy, $E_{B}$ is the binding energy of the initial state, and $C(h v)$ includes factors to account for the photon flux, escape depth, detector efficiency and reflection and refraction of the photon beam at the sample ${ }^{11},{ }^{12}$. The latter term is technically also a function of $E_{B}$, but the effect is weak and it will not significantly affect the shape of the valence band spectrum. $N_{P d}$ and $N_{M n}$ represent the fractional contribution of d electrons supplied by each element to the density of states, $D_{P d}\left(E_{B}\right)$ and $D_{M n}\left(E_{B}\right)$ are the normalised partial density of states of in the Pd $4 \mathrm{~d}$ and $\mathrm{Mn} 3 \mathrm{~d}$ respectively, and $\sigma_{P d}(h v)$ and $\sigma_{M n}(h v)$ the photoionisation cross-sections neglecting variations across the band.

Photoemission transitions from 4d or 5d metals such as Pd and Pt to particular final states produce a characteristic minimum in the cross-section, known as the Cooper minimum ${ }^{13}$ which is due to the cancellation of matrix element integrals. In the $\mathrm{Pd}_{2} \mathrm{MnIn}$ alloy, excitation energies corresponding to the region of the Pd $4 \mathrm{~d}$ Cooper minimum will enhance relatively the contribution from transitions arising from $\mathrm{Mn} 3 \mathrm{~d}$ 
derived states. In the case that the Pd $4 \mathrm{~d}$ cross-section at the Cooper minimum can be considered negligible in comparison with that for Mn 3d, then $\sigma_{X}$ in Equation (1) can be taken as zero and the spectrum will reflect the shape of the Mn 3d partial density of states, as the cross-sections of $d$ states is considerably greater than those of $\mathrm{s}$ and $\mathrm{p}$ states.

Another technique that utilises the tuneable radiation associated with a synchrotron source, may be employed to investigate the contribution from Mn 3d derived states, namely resonant photoemission. At photon energies near of the $3 p$ threshold, photoemission from a 3d state can occur either directly, or via promotion of a $\mathrm{p}$ electron to an unoccupied d state followed by autoionisation. The interference of the direct and indirect processes results in a characteristic modulation in photoemission intensity from regions of the valence band possessing Mn 3d character. The lineshape has been described by Fano ${ }^{14}$ where the modulated photocurrent, $I_{\text {mod }}\left(h v, E_{B}\right)$, can be expressed as

$$
\begin{gathered}
I_{\text {mod }}\left(h v, E_{B}\right)=I_{r}\left(h v, E_{B}\right)\left[\frac{\left\{q\left(E_{B}\right)+\varepsilon\left(h v, E_{B}\right)\right\}^{2}}{1+\varepsilon\left(h v, E_{B}\right)^{2}}\right]+I_{n r}\left(h v, E_{B}\right) \\
\text { where } \varepsilon\left(h v, E_{B}\right)=\frac{2\left[h v-h v_{0}\left(E_{B}\right)\right]}{\Gamma\left(E_{B}\right)}
\end{gathered}
$$

Here, $I_{r}\left(h v, E_{B}\right)$ and $I_{n r}\left(h v, E_{B}\right)$ represent the resonant and non-resonant photoemission intensities respectively. The parameters characterising the lineshape are the resonance energy, $h v_{0}\left(E_{B}\right)$, the asymmetry parameter, $q\left(E_{B}\right)$ and the spectral width, $\Gamma\left(E_{B}\right)$. The shape and intensity of a resonance are influenced by the degree of initial state hybridisation ${ }^{15,16,17}$ and so a quantitative analysis of resonant lineshapes can provide information on the localisation of states. 
The aim of the research described in this paper was to utilise the methods outlined above to provide information on the valence band electronic structure of the antiferromagnetic Heusler alloy $\mathrm{Pd}_{2} \mathrm{MnIn}$. In particular, the Pd 4d Cooper minimum and the Mn 3p to 3d resonance was employed to investigate the partial density of states for the Mn 3d band, since it is the Mn sites that carry the magnetic moment. The results will be discussed in the context of earlier photoemission studies of Heusler alloys and available band structure calculations.

\section{EXPERIMENTAL DETAILS}

A slug of $\mathrm{Pd}_{2} \mathrm{MnIn}$ was prepared by melting the appropriate quantities of the high purity constituent elements in an argon arc furnace. It was then annealed in an evacuated quartz ampoules at $1000 \mathrm{~K}$ for 48 hours cooled at $1 \mathrm{~K} / \mathrm{hr}$ to below the order-disorder transition temperature of $880 \mathrm{~K}$ and then cooled at $1 \mathrm{~K} / \mathrm{min}$ to room temperature; a recipe that had previously been shown to produce the $\mathrm{L} 2{ }_{1}$ structure. The structure was verified by X-ray diffraction. The slug was machined by spark erosion to a useable shape and the front face was polished to a final finish of $1 \mu \mathrm{m}$ using diamond paste. The sample was then cleaned by immersion in degreasing solvents. Cleaning in-situ was effected by scraping the surface with a diamond file mounted on a wobble stick, to ensuring that the oxidised layer was completely removed. The elemental composition of the surface was investigated by Auger electron spectroscopy before and after scraping and it was found that the cleaning process had produced a surface that was close to stoichiometric. 
Photoemission measurements were performed under conditions of ultrahigh vacuum on beamline 6 at the Synchrotron Radiation Source, Daresbury ${ }^{18}$. An angle-integrated, double-pass cylindrical mirror analyser was employed with pass energy $10 \mathrm{eV}$ to detect and energy sort the emitted electrons. The detector was aligned at $90^{\circ}$ to the incoming photon beam and photon flux was monitored by measuring the drain current from a tungsten mesh for spectrum normalisation.

Electron energy distribution curves were obtained by detecting a range of electron kinetic energies at constant photon energy. Binding energies were then referenced to the Fermi level $\left(\mathrm{E}_{\mathrm{F}}\right)$, which was assigned a value of zero. The experimental resolution in the photon energy range $40-70 \mathrm{eV}$ was previously ${ }^{19}$ estimated to be $0.2 \mathrm{eV}$ HWHM. Two types of experiments were performed: In one experiment the photoemission spectrum was collected at the Cooper minimum for Pd and at another photon energy well away from it, in the second, electron energy distribution curves were collected in small photon energy increments through the Mn 3p to 3d resonance.

\section{RESULTS AND DISCUSSION}

A typical electron energy distribution curve is shown in Figure 1(a), it was collected at a photon energy of $80 \mathrm{eV}$ where the contributions from states derived from the Pd 4d and Mn 3d will dominate the other valence states by virtue of having a much larger cross-section $^{20}$. The Fermi level is well replicated in the spectrum, but the region of highest density of states is to be found between 2 and $6 \mathrm{eV}$ below the edge. At this photon energy, the spectrum will be dominated by the full Pd 4d band as it contributes more d-electrons per formula unit than the Mn 3d and it has a slightly larger crosssection. 
Further information about the contributions of the Mn and Pd d-electrons to the photoemission spectrum can be obtained by varying the photon energy. Fig. 1(b) shows the spectrum obtained utilising a photon energy of $130 \mathrm{eV}$ which is close to the Cooper minimum ${ }^{21}$ for the Pd $4 \mathrm{~d}$. This spectrum will be dominated by the Mn 3d contribution as the cross-section for the Pd $4 \mathrm{~d}$ is very low. The spectrum is clearly different in shape with a prominent feature emerging at a binding energy of $4.7 \mathrm{eV}$, which must correspond to an Mn 3d derived state.

To facilitate comparison of the data with the calculations of Kübler et $\mathrm{al}^{6}$, we have used a procedure to extract the partial spectral weights that has been used previously $^{21}$. Taking the spectrum at $130 \mathrm{eV}$ energy to represent the partial density of states of Mn 3d, we have normalised it and subtracted it from the normalised spectrum collected with a photon energy of $80 \mathrm{eV}$. This procedure is complicated by two factors. The first is that the photoemission cross-sections will vary between 130 $\mathrm{eV}$ and $80 \mathrm{eV}$, but this was corrected for using the calculations of Yeh and Lindau ${ }^{20}$. The second complication is that the yield from the $\mathrm{W}$ grid that is used to normalise the photoemission current varies with photon energy in a way that is not well known. The correct scaling factor was determined by applying the constraint that there were $20 \mathrm{~d}$ electrons associated with the Pd 4d and 5 with the Mn 3d. The precise details of this constraint only weakly affect the resulting spectral weights.

Before background subtraction, the two spectra had the background removed using the Shirley algorithm ${ }^{22}$ starting from $6 \mathrm{eV}$ binding energy. The procedure outlined in the last paragraph was then used to determine the partial spectral weights. These partial spectral weights associated with Pd 4d and Mn 3d derived states are shown in Fig. 1(c), the Mn contribution indicated by open triangles and the Pd by filled circles. 
Both have been adjusted to give an integral of unity. Also in Fig. 1(c) is the partial density of d-states of both elements calculated by the augmented-spherical-wave formalism by from Kübler et $\mathrm{al}^{6}$ adapted to show the sum of the two spin contributions. It can be seen that there are strong similarities in the shapes of the curves. Such comparisons of spectral weights and band structure calculations can be informative, but it must be borne in mind that this does not include matrix elements effects in the photoemission transition ${ }^{23}$ or small variations in escape depth across the band. The complete overlap of the Mn 3d and Pd 4d can be seen in both calculation and experiment. Features in the experimental data will be broadened relative to the calculation by both intrinsic lifetime effects and extrinsic experimental effects ${ }^{19}$ giving an overall Gaussian half-width of around $0.5 \mathrm{eV}$. The experiment shows similar bandwidths as the calculation and with similar features. The Pd 4d contribution maximises at $2.7 \mathrm{eV}$ binding energy with a shoulder at $4 \mathrm{eV}$, both corresponding to peaks in the calculated partial density of states. The Mn 3d contribution differs in shape maximising at $2.45 \mathrm{eV}$ and showing a shoulder at $4.7 \mathrm{eV}$. Both of these features correspond to peaks that are visible in the calculation, but shifted to slightly higher binding energy. The relative intensities of calculation and empirical weights are in reasonably good agreement.

The reliability of the Copper minimum method rests on the requirement for the photoemission from all the Pd 4d states to be suppressed. However, there are several solid state effects that will inhibit this effect. Bonding and anti-bonding states in Pd may exhibit differing photon-energy dependent emission intensities ${ }^{11}$ resulting in a different depth of minimum depending on binding energy. The initial state hybridisation indicated by calculations will also reduce the magnitude of the minimum. For this reason it is informative to support the conclusions of a Cooper 
minimum analysis by using resonant photoemission. A series of electron energy distribution curves were collected in the vicinity of the Mn 3p to 3d resonance. When the photon energy is equal to the difference between the binding energies of the $3 p$ and the empty 3d states there is an enhancement of photoemission from filled $3 \mathrm{~d}$ states. Fig. 2 shows the raw curves collected for photon energies between 47 and 55 $\mathrm{eV}$. The envelope of the curves clearly demonstrates the resonant enhancement at around $50 \mathrm{eV}$, which indicates that significant $\mathrm{Mn} 3 \mathrm{~d}$ nature is retained in the alloy: the stronger the resonance the more localised and atomic-like the initial state.

It is useful to compare the curve obtained at the resonance maximum with that in the minimum before the resonance ("on and off” resonance). As the resonant photoemission pathway decays into the inelastic background and causes it to exhibit the same variation in intensity it is necessary to first remove the background using the Shirley method referred to before. To make a good comparison, it is necessary to adjust the intensity to account for variations in photoemission cross-section and yield from the $\mathrm{W}$ grid. This was done by recording the In 4d levels within the same spectra and adjusting the intensity of these to fit the calculated variation in cross-section ${ }^{20}$ with photon energy. This procedure is complicated by the maximisation in the In cross-section at around $50 \mathrm{eV}$ but appears to be reliable. The on and off resonance spectra are shown together in Fig. 3 in comparison with the Mn 3d partial density of states. It can be seen that there is a shape change between the two spectra, with the on-resonance peak showing enhancement near both the main features in the calculated curve, at 2.5 And $4.7 \mathrm{eV}$. Both of these are entirely consistent with the Mn partial spectral weight as determined using the Cooper minimum method. 
The intensity of the Mn 3p to 3d resonance may be related to the localisation of the Mn d states. Quantitative analysis of resonances in similar compounds has been used to infer atomic-like or hybridised nature of the states. For example, Robey et $a l^{24}$ analysed $\mathrm{Ni}_{2} \mathrm{MnSb}$ by resonant photoemission and associated relatively strong resonant features with weakly hybridised states. In the limit of no hybridisation, resonances associated with $\mathrm{Mn}$ 3d derived states were stated to be characterised by $q$ values in the range 2.2-2.5, as found for atomic $\mathrm{Mn}^{25}$. More strongly hybridised states were associated with weaker resonances, and gave $q$ values that were significantly lower.

To investigate the resonance in $\mathrm{Pd}_{2} \mathrm{MnIn}$ more quantitatively, a Fano analysis was performed on the resonant lineshape of the feature at $4.7 \mathrm{eV}$ binding energy. Prior to fitting, the data were carefully prepared by removing the inelastic background. The intensity from the $4.7 \mathrm{eV}$ feature was then extracted and is shown in the inset to Fig. 2. The fit to the data was then performed using Equation 2 with a linear non-resonant contribution. The Fano parameters so extracted are listed in Table 1. The resonance energy, $h v_{0}$, of $49.3 \pm 0.2 \mathrm{eV}$ is typical of such compounds. The asymmetry parameter, $q$, which has a value of $1.6 \pm 0.4$ suggests that there is a good deal of hybridisation between the bands as indicated by the calculations of Kübler ${ }^{6}$. Thus, although Heusler alloys are often quoted as model examples of systems with localised moments, the occupied Mn 3d states cannot be described as localised. 


\section{CONCLUSIONS}

The full ordered antiferromagnetic Heusler alloy $\mathrm{Pd}_{2} \mathrm{MnIn}$ has been investigated using photoelectron spectroscopy. The shape of the photoelectron spectrum is consistent with the density on states calculated by Kübler et $\mathrm{al}^{6}$. The partial spectral weights of both the Pd 4d and Mn 3d derived states has been investigated using both the Copper minimum effect and resonant photoemission and both method have been shown to be self consistent and consistent wit the calculations. Finally a fit to the Mn 3p to 3d resonance profile has shown that the occupied Mn 3d states are relatively delocalised.

\section{ACKNOWLEDGEMENTS}

Thanks are due to Kurt Ziebeck for assistance preparing the samples and to Mike Petty, Karen Bedwell and Andrew Malins for assistance with the measurements.

$1 \quad$ F. Heusler, Verh. Deutsch Phys. Gesel. 5, 219 (1903).

$2 \quad$ P.J. Webster, J. Phys. Chem. Solids 32, 1221 (1971).

3 R.A. de Groot, F.M. Mueller, P.G. van Eugen and K.H.J. Buschow, Phys. Rev. Lett. 502024 (1983).

4 A.T. Zayak, P. Entel, J. Enkouara, A. Ayvela and R.M. Mieminen, Phys. Rev. B 68132402 (2003).

5 S. Ishida, Y. Kubo, J. Ishida and S. Asano, Journal of the Physical Society of Japan 48814 (1980).

$6 \quad$ J, Kübler, A. R. Williams and C. B. Sommers, Phys. Rev. B 281745 (1983). 
7 D.J. Doherty, J. Crangle, K-U Neumann, J.G. Smith, N.K. Zayer and K.R.A. Ziebeck, J. Magn. Magn. Mater. 140-144 189 (1990).

$8 \quad$ C.M. Hurd, I. Shiozaki and S.P. McAlister, Phys. Rev. B 26701 (1982).

$9 \quad$ P.J. Webster and R.S. Tebble, Phil. Mag. 16347 (1967).

10 S. Hufner, Photoelectron Spectroscopy: Principles and Applications, 2nd edition, (SpringerVerlag, Berlin 1996).

11 G. Rossi, I. Lindau, L. Braicovich and I. Abbati, Phys. Rev. B 28, 3031 (1983).

12 B.H. Henke, Phys. Rev. A 6, 94 (1972).

13 J.W. Cooper, Phys. Rev. 128, 681 (1962).

14 U. Fano, Phys. Rev. 124, 1866 (1961).

15 L.C. Davis, Phys. Rev. B 25, 2912 (1982).

16 A. Kakizaki, K. Sugeno, T. Ishii, H. Sugawara, I. Nagakura and S. Shin, Phys. Rev. B 28, 1026 (1983).

17 J. Igarashi and T. Nakano, J. Phys. Soc. Japan 551384 (1986).

18 P. Bailey and F.M. Quinn, SERC Daresbury Laboratory Technical Memorandum DL/SCI/TM94E (1993); T.S. Turner and D. Teehan, SERC Daresbury Laboratory Technical Memorandum DL/SCI/TM85E (1985).

19 D. Brown, M.D. Crapper, K.H. Bedwell, M.T. Butterfield, S.J. Guilfoyle, A.E.R. Malins and M. Petty, Phys. Rev. B 571563 (1998).

20 J.J Yeh and I. Lindau, At. Data Nucl. Data Tables 32, 1 (1985). 
21 H. Wright, P. Weightman, P.T. Andrews, W. Folkerts, C.F.J. Flipse, G.A. Sawatzky, D Norman and H. Padmore, Phys. Rev. B 35519 (1987).

22 D.A. Shirley, Phys. Rev. B 5, 4709 (1972).

23 T-U Nahm, M Han, S-J Oh, J-H Park, J.W. Allen and S-M Chung, Phys. Rev B 518140 (1995).

24 S.W. Robey, L.T. Hudson and R.L. Kurtz, Phys. Rev. B 46, 11697 (1992).

25 E. Schmidt, H. Schroder, B. Sonntag, H. Voss and H.E. Wetzel, J. Phys. B: At. Mol. Phys. 18, 79 (1985). 


\section{TABLE}

\begin{tabular}{cc}
\hline \hline Fano Parameters & Value \\
\hline $\mathbf{h} \mathbf{v}_{\mathbf{0}}(\mathbf{e V})$ & $49.3 \pm 0.2 \mathrm{eV}$ \\
$\mathbf{q}$ & $1.6 \pm 0.4$ \\
$\Gamma(\mathbf{e V})$ & $2.1 \pm 0.5 \mathrm{eV}$ \\
\hline \hline
\end{tabular}

Table 1. Parameters obtained from the fit to $\mathrm{Pd}_{2} \mathrm{MnIn}$ photoemission data at the $\mathrm{Mn}$ $3 p$ to $3 d$ resonance.

\section{FIGURE CAPTIONS}

Figure 1. Photoemission from $\mathrm{Pd}_{2}$ MnIn. Part (a) shows the spectrum collected at 80 eV photon energy, well away from the Pd 4d Cooper minimum. Part (b) shows the spectrum collected at $130 \mathrm{eV}$ photon energy, at the Pd 4d Cooper minimum. Part (c) shows the partial spectral weights allocated to Pd 4d and Mn 3d determined using the method described in the text, compared with the densities of states adapted from reference 6. 
Figure 2. Photoemission from $\mathrm{Pd}_{2} \mathrm{MnIn}$ for photon energies in the vicinity of the $\mathrm{Mn}$ 3p to 3d resonance. The inset shows the background subtracted and normalised photoemission intensity at $4.7 \mathrm{eV}$ binding energy with a Fano profile fitted to the data.

Figure 3. Background subtracted photoemission from $\mathrm{Pd}_{2} \mathrm{MnIn}$ showing both the onresonance (filled circles) and off-resonance (open triangles) photon energies. The data are compared with the Mn 3d density of states adapted from reference 6. 
Figure 1

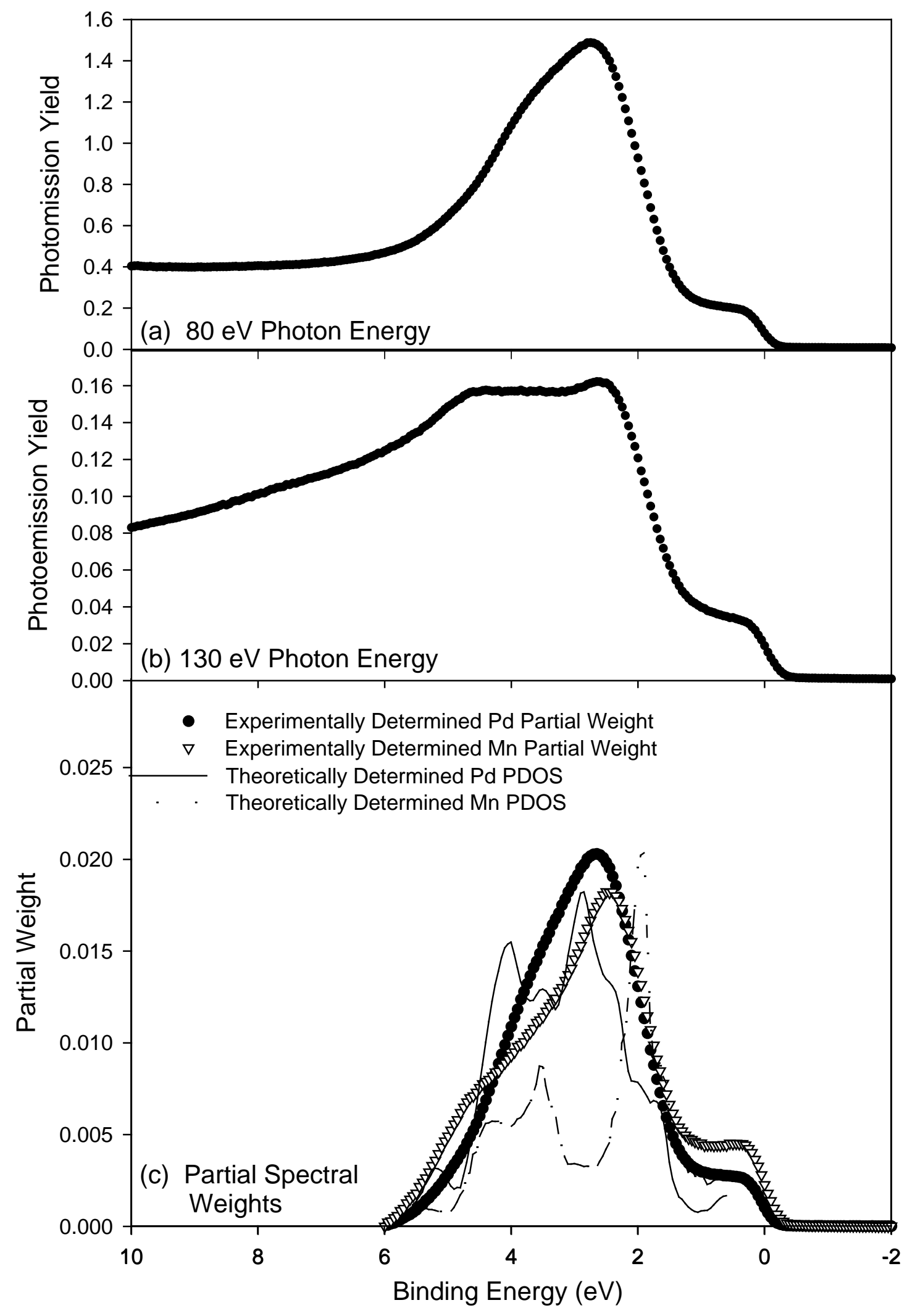


Figure 2

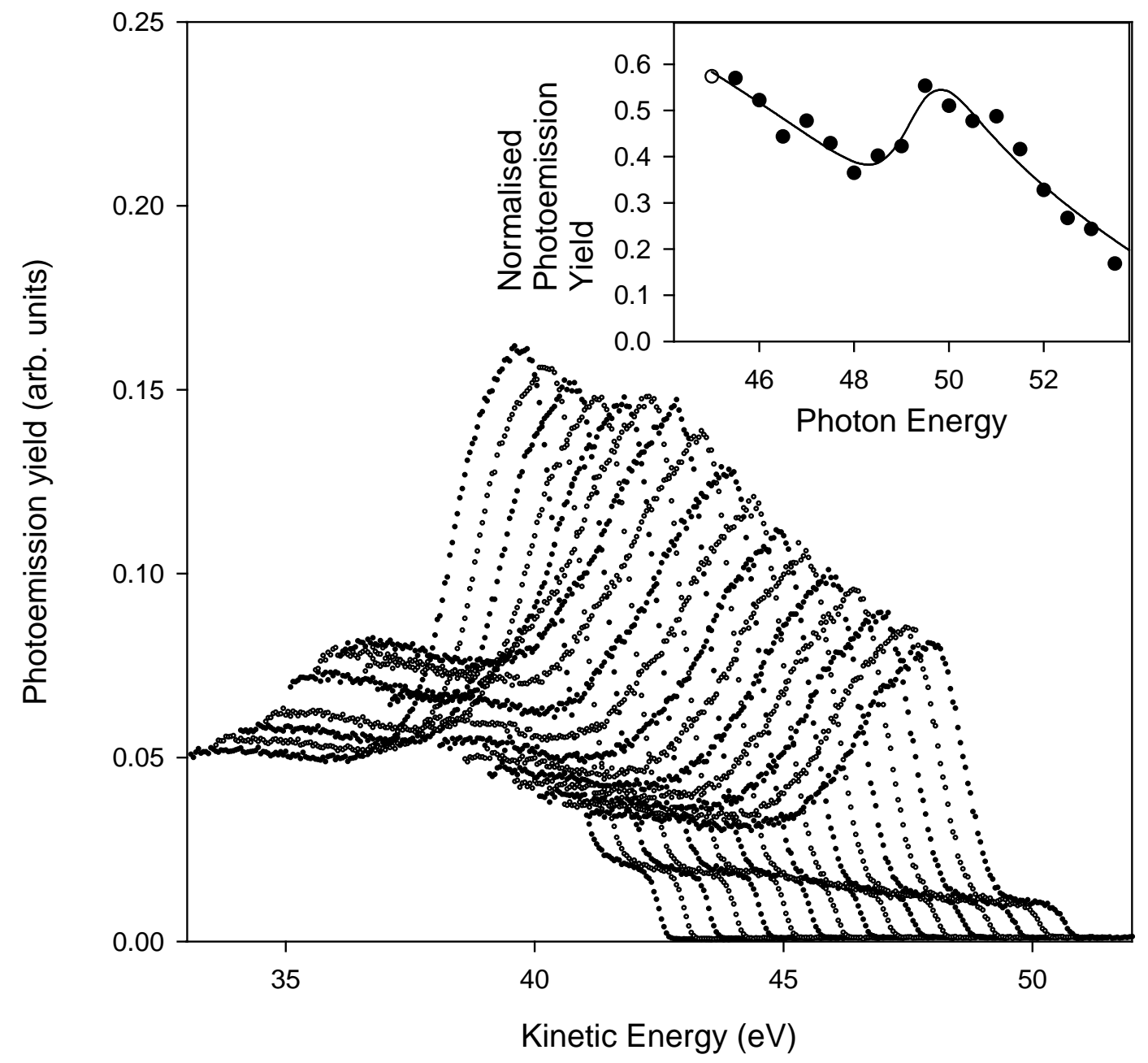


Figure 3

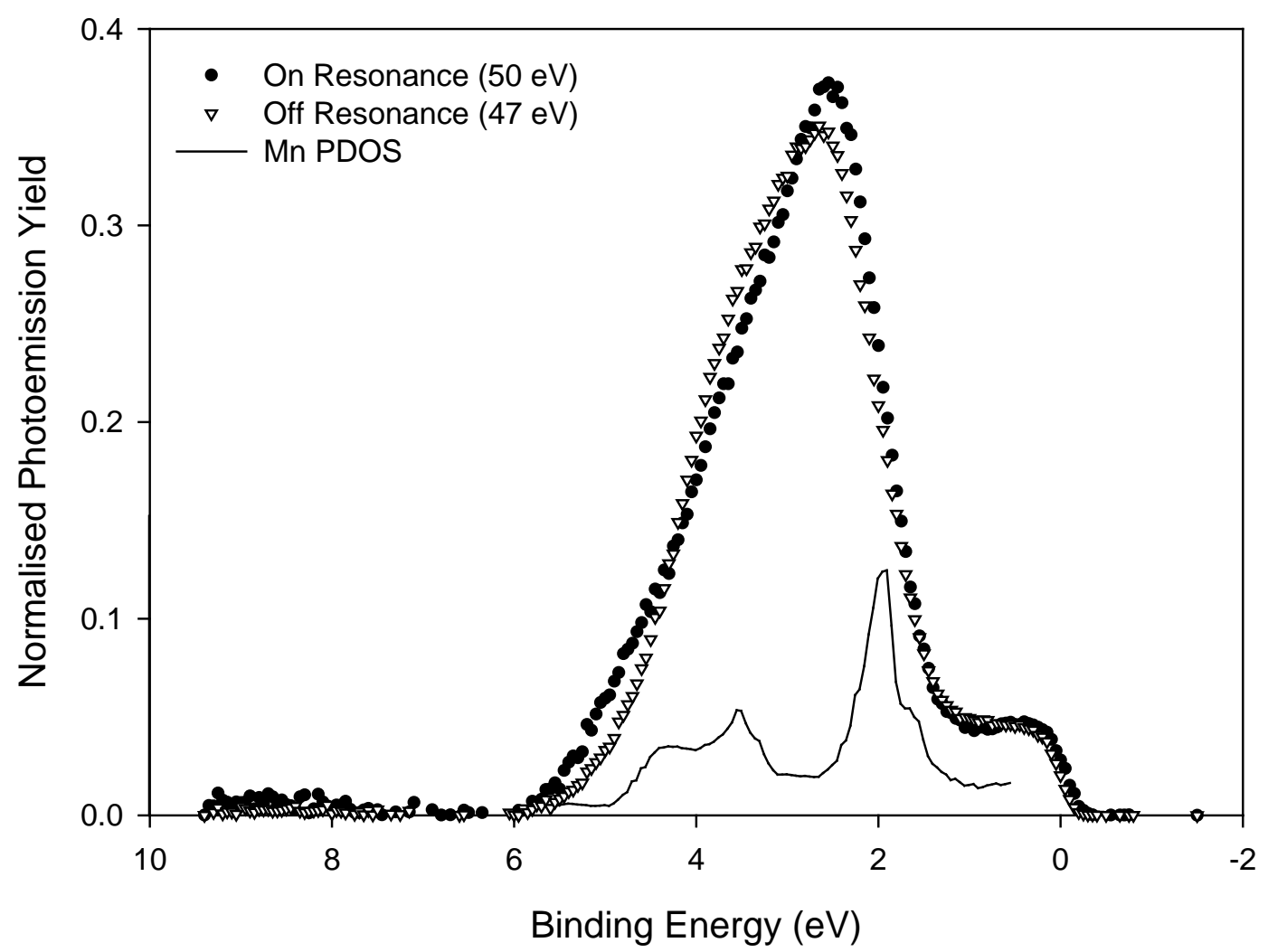

